\title{
Pattern investigation for landfill of methane gas concentration measurement
}

\begin{abstract}
One of the most important actual problems in the gas detection field is that there are strong demands for gas methane source detection to prevent explosions or poisoning accidents ...etc. In this case we need an autonomous equipment to perform wide surveying of the ground area surface based on a pattern to know its concentration on each spot of the required ground area. The best method of performing this task is to use autonomous quadcopter with high flexibility which do not affect by risk of this gas and the rough and irregular ground surface and perform the task with high speed and lower cost. This paper presents a simulation comparison of search pattern types properties and practical methane gas concentration measurement.
\end{abstract}

Keyword: Quadcopter; Methane gas; Search pattern; Sensor; Landfill 